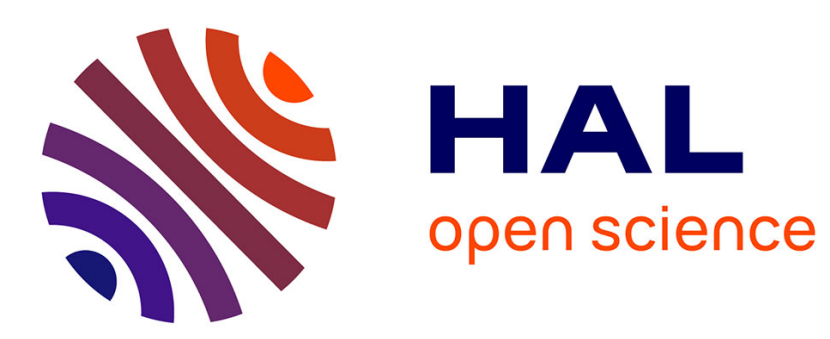

\title{
Nonlobe Reconnection at the Earth's Magnetopause for Northward IMF
}

\author{
S. Fuselier, K J Trattner, S. Petrinec, B. Lavraud, J Mukherjee
}

\section{To cite this version:}

S. Fuselier, K J Trattner, S. Petrinec, B. Lavraud, J Mukherjee. Nonlobe Reconnection at the Earth's Magnetopause for Northward IMF. Journal of Geophysical Research Space Physics, 2018, 10.1029/2018JA025435 . hal-02372780

\section{HAL Id: hal-02372780 \\ https://hal.science/hal-02372780}

Submitted on 20 Nov 2019

HAL is a multi-disciplinary open access archive for the deposit and dissemination of scientific research documents, whether they are published or not. The documents may come from teaching and research institutions in France or abroad, or from public or private research centers.
L'archive ouverte pluridisciplinaire HAL, est destinée au dépôt et à la diffusion de documents scientifiques de niveau recherche, publiés ou non, émanant des établissements d'enseignement et de recherche français ou étrangers, des laboratoires publics ou privés. 


\section{Journal of Geophysical Research: Space Physics}

\section{RESEARCH ARTICLE}

10.1029/2018JA025435

\section{Special Section:}

The Earth's Magnetosphere: New Tools, New Thinking, New Results

\section{Key Points:}

- Different magnetic field topologies at the magnetopause can be distinguished with plasma and magnetic field data

- Lobe and nonlobe reconnection occur at the magnetopause for northward IMF

- Nonlobe reconnection appears to be common at the Earth's magnetopause for northward IMF

Correspondence to:

S. A. Fuselier,

sfuselier@swri.edu

Citation:

Fuselier, S. A., Trattner, K. J., Petrinec, S. M., Lavraud, B., \& Mukherjee, J. (2018). Nonlobe reconnection at the Earth's magnetopause for northward IMF. Journal of Geophysical Research: Space Physics, 123, 8275-8291. https://doi.org/ 10.1029/2018JA025435

Received 5 MAR 2018 Accepted 21 SEP 2018 Accepted article online 27 SEP 2018 Published online 27 OCT 2018

\section{Nonlobe Reconnection at the Earth's Magnetopause for Northward IMF}

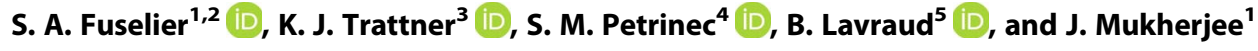 \\ ${ }^{1}$ Southwest Research Institute, San Antonio, TX, USA, ${ }^{2}$ Department of Physics and Astronomy, University of Texas at \\ San Antonio, San Antonio, TX, USA, 'aboratory for Atmospheric and Space Physics, University of Colorado, Boulder, CO, \\ USA, ${ }^{4}$ Lockheed Martin Advanced Technology Center, Palo Alto, CA, USA, Institut de Recherche en Astrophysique et \\ Planétologie, Université Paul Sabatier, Toulouse, France
}

Abstract Under northward interplanetary magnetic field conditions, magnetic reconnection at the Earth's magnetopause is usually thought to operate through the merging of magnetosheath magnetic field lines and open magnetic field lines from the magnetospheric lobe. However, reconnection also occurs between magnetosheath field lines and closed magnetic field lines in the magnetosphere. Under certain conditions, this nonlobe field line reconnection has distinct plasma and magnetic field signatures that distinguish it from reconnection of lobe field lines. A survey of these conditions suggests that nonlobe reconnection at the Earth's magnetopause may be common even for relatively strong northward IMF.

Plain Language Summary Magnetic reconnection interconnects magnetic field lines across a current sheet like that at the Earth's magnetopause or like that in the solar corona. This paper describes a type of interconnection of magnetic field lines at the magnetopause that was first reported for the solar corona. It appears that this type of reconnection is fairly common at the magnetopause. The different topology of this reconnection suggests a different type of interaction between the magnetic field lines in the solar wind and the field lines of the Earth.

\section{Introduction to Reconnection Topologies for Northward IMF}

Magnetic reconnection occurs at the Earth's magnetopause for any orientation of the interplanetary magnetic field (IMF). When the IMF is southward, reconnection occurs between the magnetosheath magnetic field and closed magnetic field lines equatorward of the Earth's magnetospheric cusps. This reconnection opens previously closed flux tubes and, through convection of the open field lines over the poles, ultimately adds magnetic flux to the Earth's magnetotail.

When the IMF is northward, reconnection is usually thought to operate through merging of magnetosheath magnetic field lines and open (i.e., lobe) magnetic field lines poleward of the cusps (e.g., Dungey, 1963; Gosling et al., 1991). Reconnection for northward IMF is common. Statistical studies show that it occurs greater than $90 \%$ of the time (Fuselier et al., 1995, 2012). Reconnection of open field lines poleward of the cusps links magnetosheath field lines with open flux tubes in the Earth's magnetospheric lobes. For strongly northward orientations, reconnection of the same magnetosheath field line may occur in both lobes poleward of the northern and southern cusps (Onsager et al., 2001; Lavraud, Thomsen, et al., 2005, Lavraud, Fedorov, et al., 2005). There is evidence that the two reconnections do not occur simultaneously (e.g., Fuselier, Petrinec, et al., 2014), but the end result is closed magnetic field lines at the dayside magnetopause that slowly convect to the nightside. In this process, the magnitude of the magnetic flux added to the Earth's magnetotail is very small compared to the amount added when the IMF is southward.

Lobe reconnection is not the only magnetic topology possible when the IMF is northward. Cowley (1983) suggested several different magnetic topologies that may result from reconnection when the IMF is northward. Two of these topologies are illustrated in Figure 1. The topology on the left-hand side results from lobe reconnection. The Sun is to the left, and a magnetosheath field line, 1, convecting toward the magnetopause reconnects in the southern hemisphere with a lobe field line, 2, poleward of the cusp. A lobe field line is defined, prior to reconnection, as a field line with one foot in the ionosphere and the other far downtail and essentially not connected to the Earth. After reconnection, the resulting open field line, $1^{\prime}$, still has one 


\section{Lobe Reconnection Poleward of the Cusp}

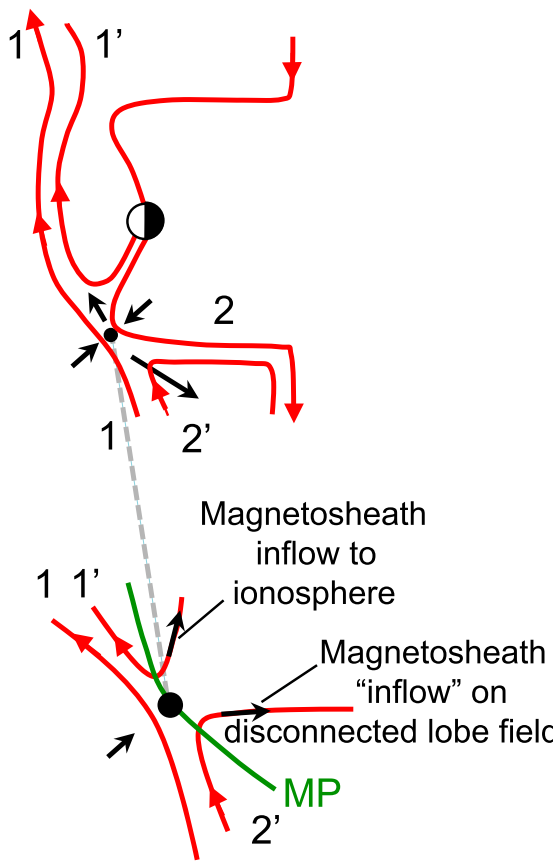

\section{Non-Lobe Reconnection Poleward of the Cusp}

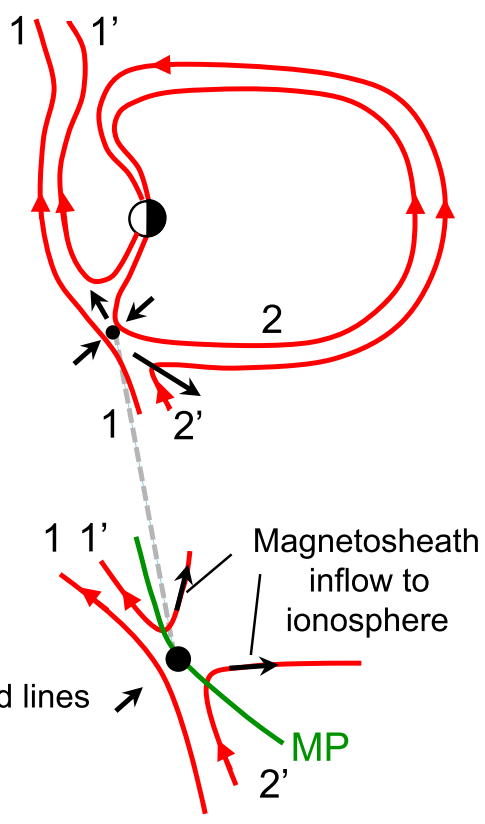

Figure 1. Two reconnection geometries for northward interplanetary magnetic field (adapted from Cowley, 1983). The lefthand panels show lobe reconnection between a magnetosheath field line (1) and an open lobe field line in the magnetosphere (2). The right-hand panels show nonlobe reconnection between a magnetosheath field line (1) and a closed field line in the magnetosphere (2). Both types of reconnection produce open field lines equatorward of the reconnection site. Poleward of the reconnection site, reconnected lobe field lines are completely open to the solar wind while nonlobe reconnection produces field lines with one foot in the northern ionosphere. The ion flows poleward of the reconnection site combined with electron signatures distinguish the two geometries.

"foot" in the ionosphere. The other end of this field line continues to convect with the solar wind and is likely to reconnect with a lobe field line in the northern hemisphere, creating a newly closed field line. Poleward of the southern cusp, the field line resulting from reconnection, $2^{\prime}$, is completely disconnected from Earth and convects tailward. The lower left-hand illustration is an enlargement of the reconnection region and shows that field line $1^{\prime}$ has plasma flowing into the cusp antiparallel to the magnetic field while field line $2^{\prime}$ has plasma flowing onto disconnected field lines in the lobe parallel to the magnetic field.

The right-hand side of Figure 1 shows a different kind of reconnection for northward IMF. This type of reconnection occurs between magnetosheath field lines and closed field lines that are poleward of the cusp. The magnetosheath field line, 1 , reconnects in the southern hemisphere creating field lines $1^{\prime}$ and $2^{\prime}$, with one foot in the southern and northern hemisphere ionosphere, respectively. The other end of field line $1^{\prime}$ convects with the solar wind and is likely to reconnect in the northern hemisphere poleward of the cusp. One end of the open field line, $2^{\prime}$, also convects tailward with the solar wind. From here on, this type of reconnection is referred to as nonlobe reconnection. This term is used here to describe reconnection at high latitudes, poleward of the cusp, between magnetosheath field lines and closed field lines on the dayside.

The lower right-hand side of Figure 1 is an enlargement of the nonlobe reconnection topology. Equatorward (sunward) of the reconnection site, the magnetic field topology resulting from nonlobe reconnection is indistinguishable from that of lobe reconnection. However, poleward (tailward) of the reconnection site, there are differences in the topologies. In particular, the field lines inside the magnetosphere are connected to the ionosphere in the northern hemisphere for nonlobe reconnection and are completely disconnected from the Earth for lobe reconnection. There have been simulations (Vennerstrom et al., 2005; Watanabe et al., 2017) and observations (Onsager et al., 2001) arguing for nonlobe reconnection for northward IMF. 
Reconnection in the opposite hemisphere (the northern hemisphere in Figure 1) greatly complicates the topology at the magnetopause because it can occur before or after the reconnection in the southern hemisphere and can be lobe or nonlobe reconnection. The common occurrence of reconnection in both hemispheres, though not simultaneous, insures that complicated field topologies exist essentially all the time. These complicated topologies have implications for the electrons observed sunward of the magnetopause because they are able to move rapidly from one hemisphere to another. However, for the most part, the slower moving ions provide information on the local reconnection conditions, provided the magnetopause crossing occurs sufficiently far away from the opposite hemisphere (e.g., Fuselier, Frahm, et al., 2014).

When combined with electron observations and magnetic field signatures in the vicinity of one of the cusps, these ion observations distinguish lobe and nonlobe reconnection independent of the type of reconnection in the opposite cusp. These combined particle and field signatures are exploited here to distinguish lobe and nonlobe reconnection for northward IMF. Section 2 describes the Cluster instrumentation used in this study and how it is used to distinguish the type of reconnection. Section 3 describes two southern hemisphere, high-latitude magnetopause crossings under northward IMF conditions, one crossing where lobe reconnection was taking place and one crossing where nonlobe reconnection was taking place. This section provides the observations interleaved with interpretations because it is important to build the case for lobe or nonlobe reconnection in a piecemeal fashion. At the end of the section, after the cases have been built, a summary of the observations and their interpretation is provided to compare and contrast the two events. Section 4 describes results of a survey of high-latitude magnetopause crossings by the Cluster spacecraft. With some important caveats, this survey provides some indication how often nonlobe reconnection occurs at the dayside magnetopause under northward IMF. Section 5 summarizes this work.

\section{Instrumentation}

Electron, ion, and magnetic field observations from the Cluster spacecraft are used here to identify signatures of nonlobe reconnection and distinguish these signatures from lobe reconnection.

Electron observations in this paper are from the Plasma Electron and Current Experiment analyzers (Johnstone et al., 1997; Szita et al., 2001). These analyzers measure a full 3-D distribution over a combined energy range from $0.6 \mathrm{eV}$ to $26 \mathrm{keV}$ and with a maximum time resolution of $4 \mathrm{~s}$ (one spacecraft spin). Electron observations are used in combination with magnetic field data from the Cluster magnetometer experiment (Balogh et al., 1997) to determine streaming directions of suprathermal electrons in the magnetosheath boundary layer (MSBL) and the cusp. The MSBL is the reconnection layer adjacent to the magnetopause on the magnetosheath side. Heated, streaming suprathermal electrons in the MSBL are a unique signature of reconnection and open field line topologies at the magnetopause. Magnetosheath electrons are heated at the current layer and stream along open field lines into the MSBL and into the cusp/lobe. The heated electrons stream from the reconnection site along the magnetic field. When the Cluster spacecraft is between two reconnection sites, it observes bidirectional streaming electrons in the MSBL. For northward IMF, the direction of dominant streaming also often indicates which hemisphere reconnected first (Lavraud, Thomsen, et al., 2005; Fuselier, Petrinec, et al., 2014), although, as will be shown in the next section, there are other interpretations of this dominant streaming direction. Inside the magnetopause and in the magnetospheric cusps, bidirectional streaming electrons are often observed. These streaming electrons indicate that the spacecraft is either on closed field lines or on open field lines between a heating source (the magnetopause current layer) and an ionospheric mirror point. Thus, a great deal of information on the topologies of field lines in the magnetosheath and in the cusp near the magnetopause is inferred from the presence of streaming electrons and their streaming directions. Streaming electrons have been used to infer the topology of field lines near the Earth's magnetopause (Fuselier et al., 1995, 1997, 2012; Fuselier, Petrinec, et al., 2014; Lavraud, Thomsen, et al., 2005; Lavraud et al., 2006; Onsager et al., 2001; Zhong et al., 2013) and at the magnetopauses of Jupiter and Saturn (Badman et al., 2013; Ebert et al., 2017; Fuselier, Frahm, et al., 2014; McAndrews et al., 2008).

Ion observations in this paper are from the Cluster lon Spectrometer Hot lon Analyzer and Composition and Distribution Function Analyzer (Reme et al., 2001). These analyzers measure a full 3-D ion distribution over an energy range from about $5 \mathrm{eV}$ to $32 \mathrm{keV}$ (Hot lon Analyzer) and to $40 \mathrm{keV}$ (Composition and Distribution Function Analyzer) with a maximum time resolution of $4 \mathrm{~s}$. The ion velocity moments are used to 
determine where the spacecraft crosses the magnetopause relative to the reconnection location. In addition to ion moments, 3-D ion distribution functions are used here in conjunction with the electron observations to determine magnetic field topology and reconnection entry locations. Ion distributions in the cusp have been used previously to determine entry times and locations (Fuselier, Petrinec, et al., 2014).

Solar wind observations are from the Advanced Composition Explorer and Wind spacecraft. Solar wind plasma measurements (McComas et al., 1998; Ogilvie et al., 1995) are used to determine convection times from upstream spacecraft to the magnetopause, and solar wind magnetic field measurements (Lepping et al., 1995; Smith et al., 1998) are used to determine IMF clock angles. The IMF clock angles are used in a model (see Trattner et al., 2007) to predict the magnetic shear at the magnetopause and the location of antiparallel reconnection sites (see Fuselier, Petrinec, et al., 2014).

\section{Observations}

\subsection{March 2004: Lobe Reconnection}

Figure 2 shows Cluster 3 observations of a crossing poleward of the southern cusp on 10 March 2004. The panels from top to bottom are (a) the omnidirectional ion flux, (b) the ion density, (c) the ion temperature, ( $d$ and e) the ion velocities in GSM coordinates and parallel and perpendicular to the magnetic field, ( $f$ ) the three components and magnitude of the magnetic field, and ( $g$ and $h$ ) the electron fluxes parallel and antiparallel to the magnetic field. The spacecraft is in the magnetosheath and crosses the magnetopause first at 1226 UT (shown by the solid vertical line), then makes several partial crossings (shown by vertical dashed lines), and finally, makes two additional full crossings before entering the magnetosphere/lobe after 1233:30 UT.

Across the magnetopause current layer, the magnetic field in (f) rotates from positive $B_{Z}$ in the magnetosheath/MSBL to negative $B_{Z}$ in the magnetosphere/lobe. This rotation indicates that the spacecraft crossed the magnetopause poleward of the southern cusp. For crossings equatorward of the cusp, the $B_{Z}$ component would remain positive across the magnetopause. All of the current layer crossings in Figure 2 are poleward of the southern cusp because they all show rotation from $B_{Z}$ positive in the magnetosheath/MSBL to $B_{Z}$ negative in the magnetosphere/lobe.

In the magnetosheath/MSBL, $(\mathrm{g})$ and $(\mathrm{h})$ show that there are periods of high and low fluxes of electrons with energies from about 0.4 up to $2 \mathrm{keV}$. Low fluxes both parallel and antiparallel to the magnetic field, for example, at 1223 UT, are times when the spacecraft is in the magnetosheath and not magnetically connected to any magnetopause reconnection site. High fluxes are times when the spacecraft is in the MSBL and is observing either heated, unidirectional streaming magnetosheath electrons on open field lines or heated, bidirectional streaming electrons on closed field lines. Bidirectional streaming, for example, from 1221 to 1222 UT, indicates that the spacecraft is on magnetosheath field lines that have likely reconnected poleward of both the southern and northern cusps.

Figure 3 compares electron fluxes parallel and antiparallel to the magnetic field for two time periods in the MSBL with fluxes in the magnetosheath (these time periods are identified by the blue triangles in Figure 2 g). The panel on the left in Figure 3 compares a time period when unidirectional streaming electrons were present in the MSBL with one in the magnetosheath. The parallel propagating populations in the MSBL and magnetosheath are nearly the same. However, the antiparallel propagating population in the MSBL has an extra population of higher energy streaming electrons that originate from open field lines at the magnetopause. The panel on the right in Figure 3 compares a time period when bidirectional streaming electrons were present in the MSBL with one in the magnetosheath. In this panel, only the lowest energy populations in the MSBL and magnetosheath are similar. Both the parallel and antiparallel populations in the MSBL are dominated by streaming electrons that originate from the open field lines associated with two reconnection sites poleward and equatorward of the spacecraft.

Bidirectional streaming is nearly always observed at high latitudes and indicates that this type of double reconnection is common (Lavraud, Thomsen, et al., 2005; Lavraud et al., 2006; Fuselier et al., 2012). There are also bursts of unidirectional streaming electrons, for example, in the time period from 1222 to 1223:30 UT in Figure 2. The predominantly antiparallel direction of this streaming has at least two interpretations. In the first interpretation, the spacecraft is in the MSBL poleward/southward of the southern hemisphere 


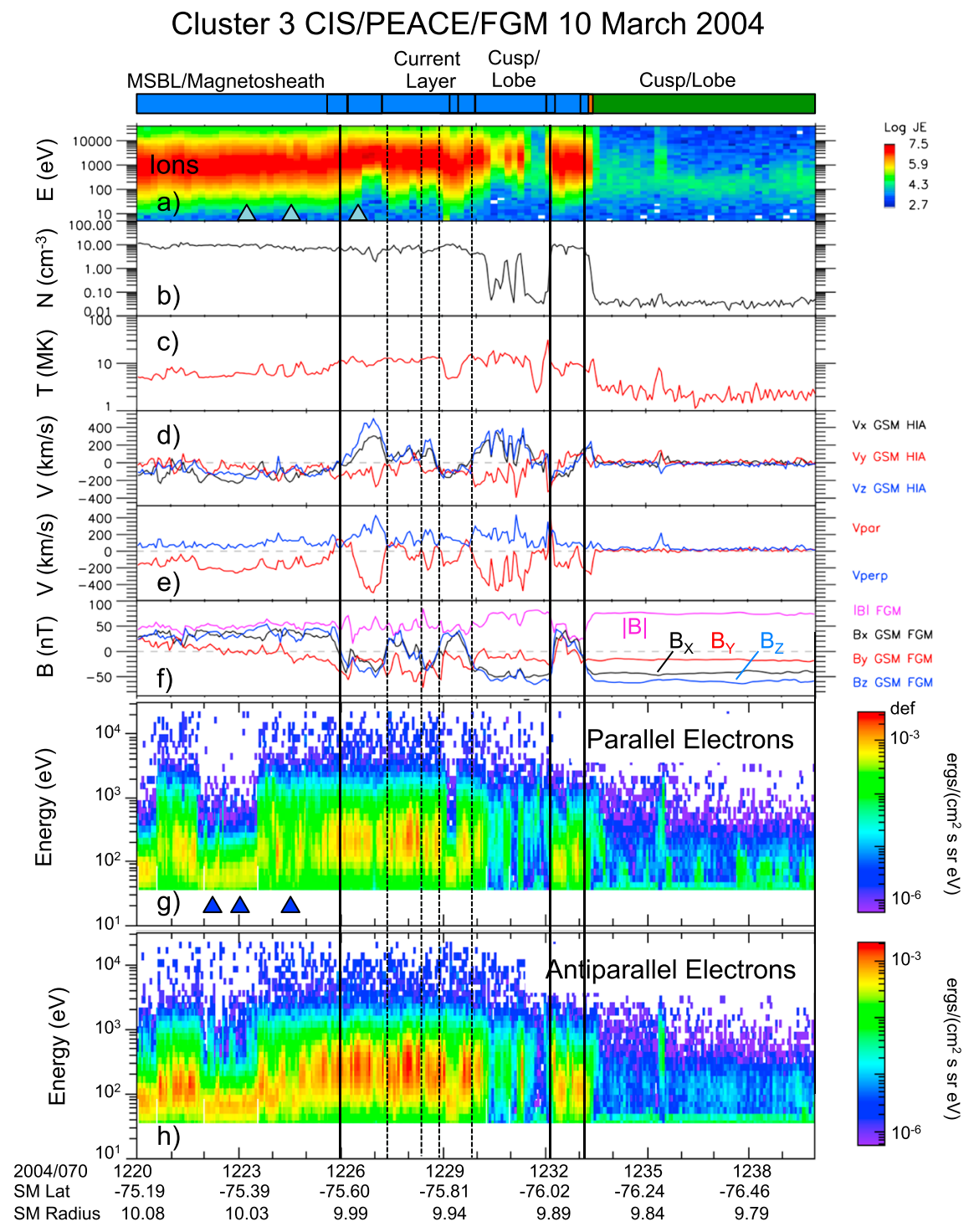

Figure 2. Cluster 3 observations during a series of magnetopause crossings where lobe reconnection is occurring. The regions are identified by the bars at the top. From top to bottom are the omnidirectional ion flux, the ion density and temperature, the ion velocities in geocentric solar magnetospheric (GSM) and field aligned coordinates, the magnetic field in GSM, and the electron fluxes parallel and antiparallel to the magnetic field. Partial crossings of the magnetopause current layer are identified by the vertical dashed lines, and full crossings are identified by vertical solid lines. (a) The gray triangles show the times of three ion distribution functions in Figure 4. The magnetic field rotates from northward in the magnetosheath to southward in the magnetosphere. The southward field direction in the magnetosphere indicates that the spacecraft crosses poleward of the southern magnetospheric cusp. Bidirectional heated, streaming electrons in the magnetosheath boundary layer indicate that reconnection is occurring in both hemispheres, probably poleward of both the northern and southern cusps. The high ion velocities in the cusp that are primarily antiparallel to the magnetic field, for example, at 12:27 UT are magnetosheath ions that have crossed the magnetopause and are propagating toward the southern ionosphere. The propagation direction and the bidirectional streaming electrons indicate that the spacecraft crossed the magnetopause equatorward of the high-latitude reconnection site. The last two crossings at 1232 UT and 1233 UT are different. Inside the magnetosphere, there are low ion fluxes at $\sim 1 \mathrm{keV}$ and low electron fluxes. The low fluxes indicate that the spacecraft is in the lobe, probably on field lines that are completely open.

high-latitude reconnection site and observes antiparallel streaming electrons from that site only. In the second interpretation, the reconnection site northward of the spacecraft (probably all the way poleward of the northern cusp) reconnects first as the field line drapes against the magnetopause and the spacecraft is observing streaming electrons from the northern hemisphere site only. In the first interpretation, the 


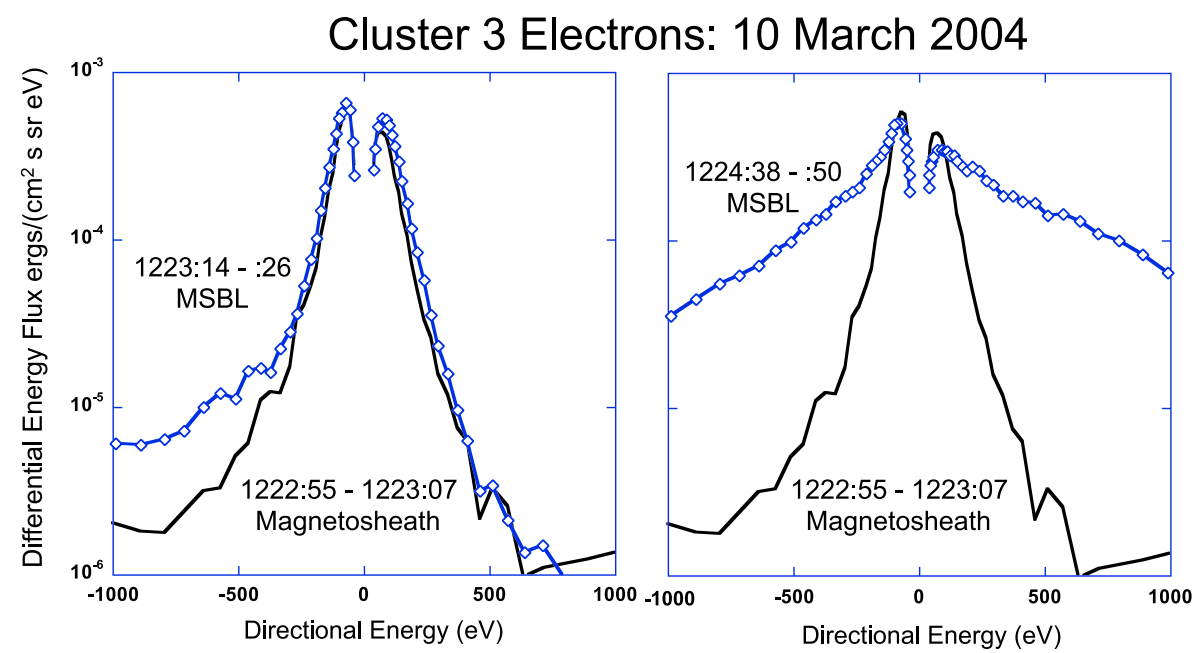

Figure 3. Electron distributions in the magnetosheath boundary layer (MSBL) and magnetosheath. (left) A population of unidirectional electrons in the MSBL is streaming antiparallel to the magnetic field, while the populations parallel to the magnetic field in the MSBL and magnetosheath are very similar. (right) Bidirectional streaming electrons in the MSBL dominate the distribution at nearly all energies, and the distributions in the MSBL and magnetosheath are similar only at the lowest energies.

alternation between unidirectional, anti parallel streaming, and bidirectional streaming may indicate that the spacecraft is very close to the southern hemisphere reconnection site. In the second interpretation, the distance to either the northern or the southern hemisphere reconnection site is not known.

Across the magnetopause current layer and in the cusp, there are several time periods when the heated, bidirectional streaming electrons persist. In these periods, the spacecraft is likely on field lines that are either open with one foot in the ionosphere or are closed with foot points in both the northern and southern highlatitude ionosphere. Given that reconnection may not only occur first in the northern hemisphere but also occur sometime later in the southern hemisphere, it is possible that these periods where the heated, bidirectional streaming electrons persist are times when the spacecraft is on recently closed field lines. On these field lines, the spacecraft observes electrons that entered the cusp in both the northern and southern hemispheres and are mirroring in both ionospheres.

The final two crossings of the current layer, at 1232 and 1233 UT in Figure 2, are different from the earlier crossings. In particular, these two crossings show different electron signatures inside the magnetosphere. At the current layer crossing at 1233 UT, antiparallel and parallel streaming electrons persist as the field rotates in the current layer. However, after that rotation is complete, the parallel streaming electrons persist briefly and then the spacecraft is on field lines with only low fluxes of low-energy electrons. There are bursts of low-energy electrons primarily in the parallel direction and one brief burst of bidirectional streaming higher-energy electrons at 1235:30 UT. The absence of significant fluxes of bidirectional streaming electrons in the energy ranges from a few tens of $\mathrm{eV}$ to several $\mathrm{keV}$, and a similar absence of significant fluxes of magnetosheath ions at $\sim 1 \mathrm{keV}$ in (a) indicates that the spacecraft was mostly on lobe field lines from 1233 UT to 1240 UT. The lower energy, predominantly parallel streaming electrons observed at 1238 UT, for example, are likely magnetosheath electrons that enter onto field lines that are completely disconnected from the ionosphere, as illustrated by field line $2^{\prime}$ in Figure 1, lower left-hand panel.

Figures $2 \mathrm{~d}$ and 2 e show ion flow velocities. In the magnetosheath and MSBL, the flow velocities in GSM coordinates in (d) show that the ions have very little velocity in the $Y$ direction and the velocities in the $X$ and $Z$ directions are mostly negative. Given the position of the spacecraft in the southern hemisphere, the negative $X$ and $Z$ velocities are consistent with flow around the magnetospheric obstacle. The velocities in (e) in fieldaligned coordinates show that the magnetosheath flow is primarily antiparallel to the magnetic field, again consistent with flow around the magnetosphere in the northward IMF-dominated magnetosheath. The flow in the MSBL, for example, from 1221 to 1222 UT, is lower than the flow in the magnetosheath, for example, at 

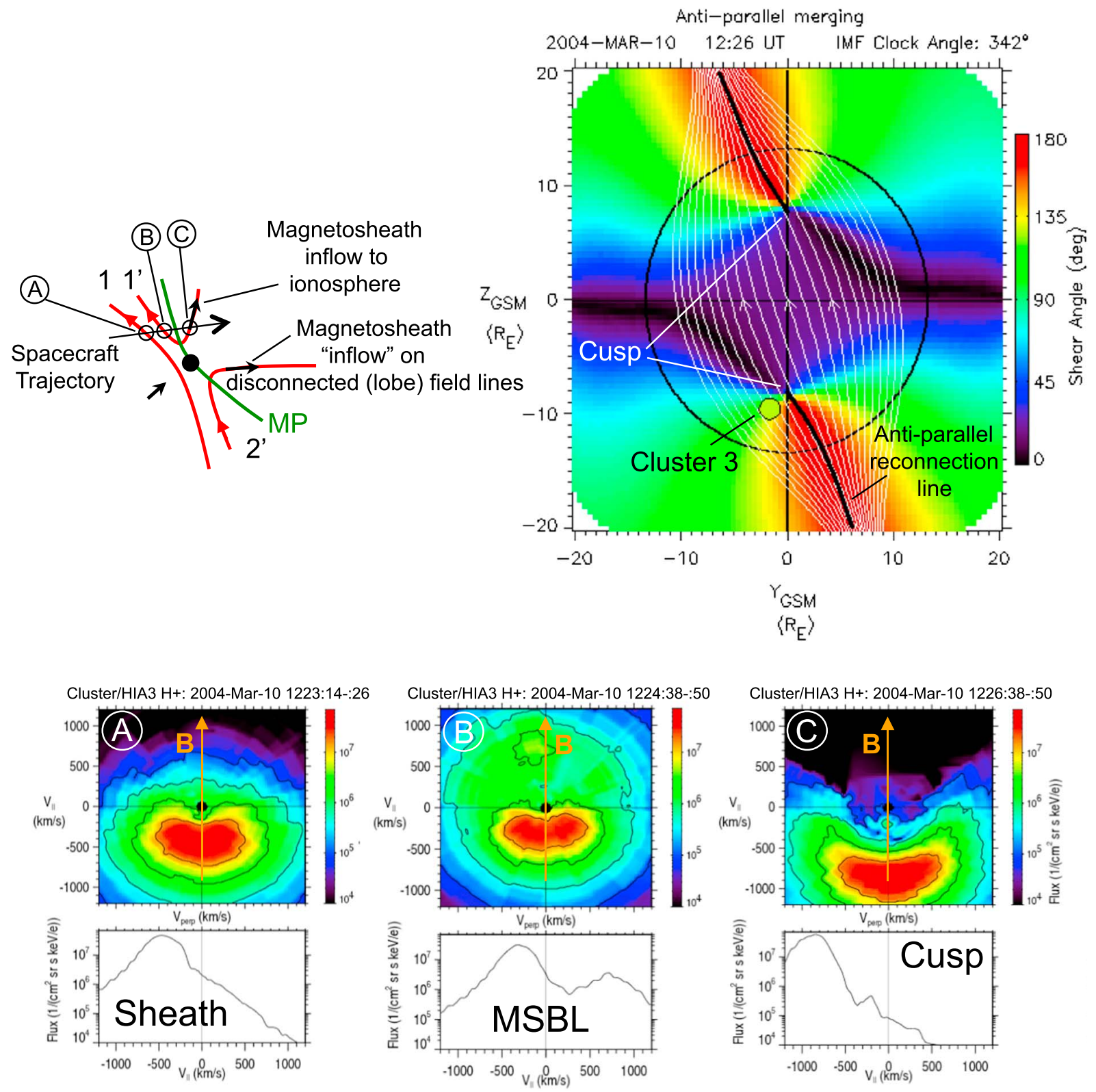

Figure 4. (upper left-hand panel) Schematic of the magnetopause crossing showing the location of the three ion distributions in the bottom panels. (upper righthand panel) $Y-Z$ geocentric solar magnetospheric projection of the shear angles at the magnetopause showing the location of the Cluster spacecraft poleward of the southern cusp. The spacecraft crosses the magnetopause equatorward of the southern reconnection site. In the bottom panels, the magnetosheath population is flowing antiparallel to the magnetic field, in the direction around the magnetosphere. This population is seen in the magnetosheath boundary layer along with a high-speed population propagating parallel to the field. The parallel-propagating population is from the reconnection site that is poleward of the spacecraft. In the cusp, the high-speed population is propagating toward the southern ionosphere.

1223 UT. An explanation for this reduction in the flow speed is provided below. In the cusp, for example, centered on 1227 UT, there are fast sunward/northward flows. Panel e shows that these fast flows are antiparallel to the magnetic field. Since the magnetic field reversed across the magnetopause, the antiparallel flows are directed toward the ionosphere along reconnected field lines from the southern cusp.

The lower panels of Figure 4 show ion distributions in (a) the magnetosheath, (b) MSBL, and (c) cusp for the crossing at 1226 UT in Figure 2. The distributions are 2-D slices in the $V_{\|}-V_{\perp}$ plane in the frame where the bulk velocity perpendicular to the magnetic field direction is zero. Below each 2-D distribution is a 1-D cut along the $V_{\|}$direction. 
In the magnetosheath (a) a single ion population streams antiparallel to the magnetic field (i.e., around the magnetospheric obstacle). This same magnetosheath population is in the MSBL (b along with a second population that is streaming parallel to the magnetic field. The parallel-streaming population is a combination of "reflected" ions (Cowley, 1982; Fuselier et al., 1991) and ions that have "leaked" out of the magnetosphere on open field lines and are streaming away from the reconnection site that is southward/tailward of the spacecraft. The flow velocity of the combined populations in the MSBL is lower than the flow velocity of the single magnetosheath population. This combination explains the lower antiparallel velocities in the MSBL in Figure 2e) when compared to the velocities in the magnetosheath. The MSBL distribution (b) is observed during a period of bidirectional streaming electrons, possibly from both the northern and southern reconnection sites. Accepting this interpretation of bidirectional streaming electrons, no antiparallel streaming ions are observed at higher velocities in the MSBL because the northern reconnection site is too far away from the spacecraft. Thus, even though the electron observations suggest that the northern hemisphere reconnection occurred first, there was not sufficient time for the slower ions to arrive in the southern hemisphere. The cusp distribution (c) in Figure 4 shows a single, fast, antiparallel streaming magnetosheath population. These are the magnetosheath ions that crossed the magnetopause current layer and are propagating toward the ionosphere. The bend in the distribution indicates that these ions entered the cusp at a location where the magnetic field strength is lower and that they are conserving their first adiabatic invariant as they propagate into a cusp region where the field strength is higher (see, e.g., Fuselier, Petrinec, et al., 2014; Broll et al., 2017). Bidirectional streaming electrons from both hemispheres or from the magnetosheath and the southern ionosphere are observed at time (c). However, the ions are streaming in one direction (toward the ionosphere) because there was insufficient time after reconnection to allow the ions to travel to the ionosphere, mirror, and return to the spacecraft.

The upper left-hand panel of Figure 4 shows the field topology and the spacecraft trajectory through the reconnected field lines for the three distributions in the lower panels. The upper right-hand panel shows the magnetic shear at the magnetopause for the crossing at 1226 UT. These shear angles were computed using modeled, draped magnetosheath (in white in Figure 4) and magnetospheric field lines as described previously (e.g., Fuselier, Frahm, et al., 2014; Trattner et al., 2007). They show that the spacecraft was at high latitudes just poleward of the cusp when it crossed the reconnected field lines. The right-hand panel also shows that the antiparallel reconnection site in the southern hemisphere is relatively far from the spacecraft along the draped magnetic field.

In summary, the plasma and magnetic field data in Figures 2-4 illustrate a classic encounter of lobe reconnection between magnetosheath and previously open (lobe) field lines. The observations provide the location of the magnetopause crossings relative to the reconnection sites and the topology of the field lines. The magnetic field, electrons, and ions play complementary roles in the interpretation of the magnetopause crossings in Figure 2. The magnetic field indicates the location of the spacecraft crossing relative to the cusp. The electrons indicate that multiple reconnection was occurring and indicate two possible interpretations where this reconnection is occurring. They also indicate that the spacecraft was occasionally on lobe field lines in the magnetosphere. The ions help resolve which interpretation from the electrons is more likely.

The magnetic field rotations from north to south across the magnetopause indicate that the spacecraft always crossed the magnetopause poleward/tailward of the magnetospheric cusp. Bidirectional streaming electrons in the MSBL indicate that reconnection was likely occurring poleward of both cusps (i.e., dual-lobe reconnection was occurring at the high-latitude magnetopause). The unidirectional, predominately antiparallel streaming electrons in the MSBL are interpreted one of two ways: First, that the spacecraft was occasionally on field lines connected to a southern hemisphere reconnection site that was equatorward/sunward of the spacecraft. Second, that reconnection was occurring in the northern hemisphere first (probably poleward of the northern cusp) and later reconnection occurs poleward of the southern cusp to form closed field lines. For several intervals in the cusp, bidirectional streaming electrons and antiparallel streaming ions indicate that the spacecraft crossed equatorward/sunward of the reconnection site in the southern hemisphere, supporting the first interpretation of the unidirectional streaming electrons in the MSBL. Occasionally, especially for the last two full crossings in Figure 2, the spacecraft observes low-energy, parallel streaming electrons. These are times when the spacecraft is on lobe field lines and indicates that lobe reconnection was occurring in the southern hemisphere. 


\subsection{November 2005: Nonlobe Reconnection}

Figure 5 shows Cluster 3 observations of a crossing poleward of the southern cusp on 9 November 2005. The format is the same as that in Figure 2. The spacecraft starts in the cusp/magnetosphere at 1340 UT and, after eight partial and full magnetopause crossings (identified by vertical solid and dashed lines, respectively), returns to the magnetosphere at 1353 UT.

Across the current layer, for example, from 1342:30 to 1344:30 UT, the magnetic field rotates from negative $B_{Z}$ in the magnetosphere/cusp to positive $B_{Z}$ in the magnetosheath/MSBL. This rotation is similar to the rotation across the current layers in Figure 2 and indicates that the spacecraft crosses the magnetopause poleward of the southern cusp.

Also similar to the observations in Figure 2, Figures $5 \mathrm{~g}$ and $5 \mathrm{~h}$ show that there are many periods in the cusp and MSBL where heated, bidirectional streaming electrons are observed and some intervals in the MSBL where unidirectional streaming electrons are observed. In fact, the only time periods when there are no heated electrons are briefly around 1343:30 UT and from 1351:30 to 1352:30 UT. For these two intervals, the spacecraft is in the magnetosheath and not connected to any reconnection site. Similar to Figure 2, the unidirectional streaming in the MSBL in Figure 5 is predominately in the antiparallel direction, for example, near 1348 UT and 1350:30 UT.

Figure 6, analogous to Figure 3, compares electron fluxes parallel and antiparallel to the magnetic field for two time periods in the MSBL with fluxes in the magnetosheath (these time periods are identified by the blue triangles in Figure $4 \mathrm{~g}$ ). The panel on the left in Figure 6 compares a time period when unidirectional streaming electrons were present in the MSBL with one in the magnetosheath. The parallel propagating populations in the MSBL and magnetosheath are nearly the same except at the highest energies, where the differences are due to counting statistics. However, the antiparallel propagating population in the MSBL has an extra population of higher energy streaming electrons that originate from open field lines at the magnetopause. The panel on the right in Figure 6 compares a time period when bidirectional streaming electrons were present in the MSBL with one in the magnetosheath. In this panel, only the lowest energy populations in the MSBL and magnetosheath are similar. Both the parallel and antiparallel populations in the MSBL are dominated by streaming electrons that originate from the open field lines associated with two reconnection sites poleward and equatorward of the spacecraft.

As in the other event, the unidirectional streaming direction in Figures 5 and 6 is interpreted in at least two ways. In the first interpretation, the spacecraft is poleward/tailward of a nearby southern hemisphere reconnection site (e.g., the upper left-hand sketch in Figure 7) and second, the reconnection site in the northern hemisphere reconnected first. Bidirectional streaming in the MSBL occurs when the spacecraft is between two reconnection sites, and these sites are likely poleward of the northern hemisphere and southern hemisphere cusps. Inside the magnetosphere, heated, bidirectional streaming electrons are always observed. In Figure 5, there are no intervals where the spacecraft is on lobe field lines, similar to the lobe intervals in Figure 2. Using only the electron and magnetic field observations in Figure 5, it is not possible to determine if the spacecraft crosses from the magnetosheath onto reconnected field lines equatorward/sunward of a southern hemisphere reconnection site or if the spacecraft crosses onto nonlobe reconnected field lines poleward/tailward of a southern hemisphere reconnection site.

The ion flows in Figures $5 \mathrm{~d}$ and $5 \mathrm{e}$ distinguish these two different crossing locations and magnetic field topologies. In (d) the magnetosheath flow, for example, from 1349:15 to 1353 UT, is tailward and poleward, again consistent with flow around the magnetosphere. However, the flow in the cusp is opposite to that of the cusp intervals in Figure 2. In particular, the cusp flows in Figure 5, for example, from 1342 to 1343 UT, are poleward/tailward in (d) and parallel to the field in (e). Parallel flows in the cusp are consistent with a spacecraft crossing poleward/tailward of a reconnection site in the southern hemisphere. A crossing equatorward/sunward of the reconnection site would show antiparallel flows like those in Figure 2.

The lower panels of Figure 7 show ion distributions in (a) the magnetosheath, (b) MSBL, and (c) cusp for the crossing at 1343 UT in Figure 5. These distributions illustrate the ion flows in the MSBL and cusp. The format and ordering are the same as in Figure 4 even though the spacecraft crosses from the magnetosphere to the magnetosheath (see the upper left-hand panel of Figure 7). 
Cluster 3 CIS/PEACE/FGM 9 November 2005

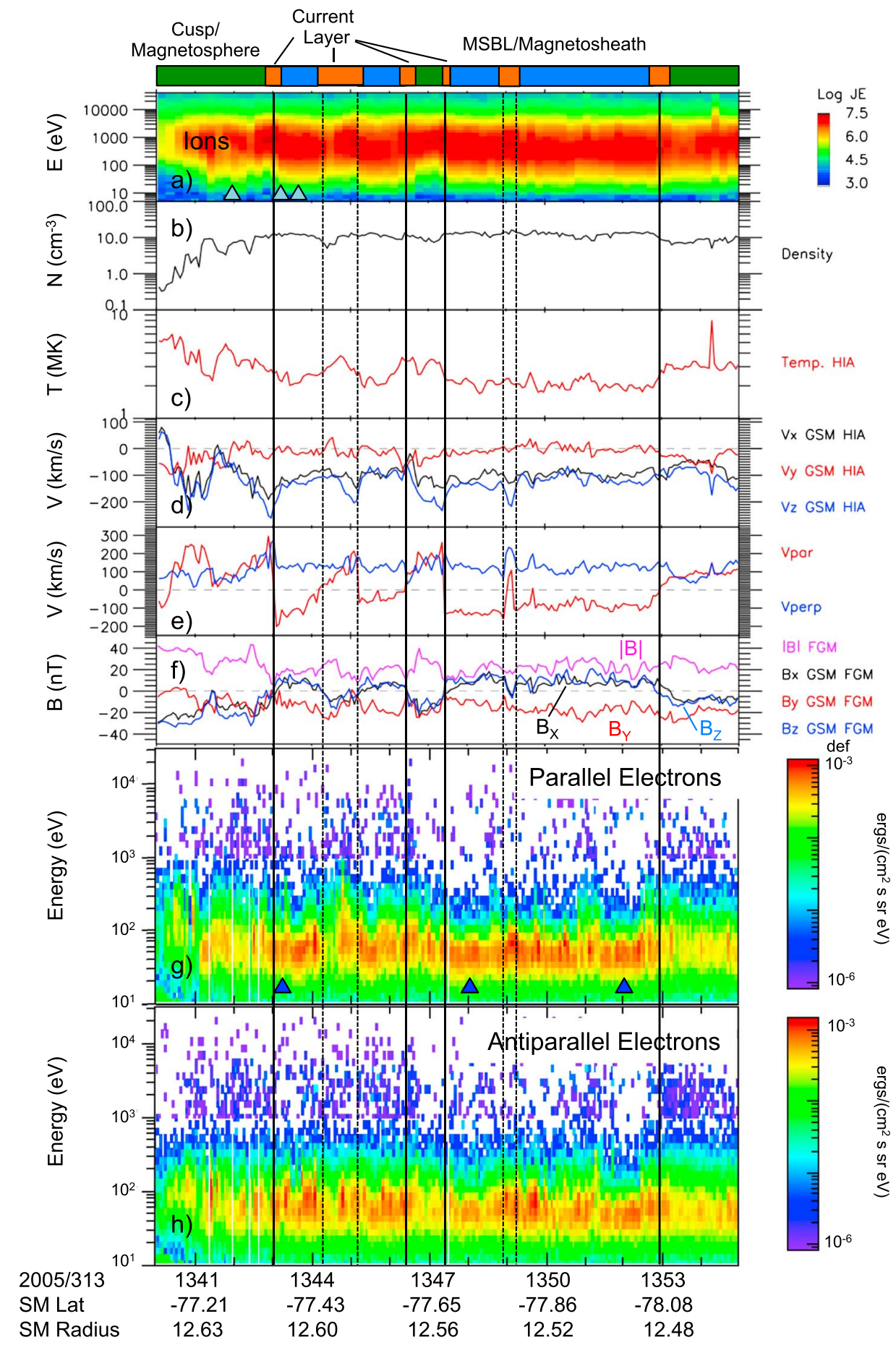

Figure 5. Cluster 3 observations during a series of magnetopause crossings where nonlobe reconnection is occurring. The format is the same as in Figure 2 . The magnetic field rotates from northward in the magnetosheath to southward in the magnetosphere, for example, from 1352 UT to 1354 UT. The southward field direction in the magnetosphere indicates that the spacecraft crosses poleward of the southern magnetospheric cusp. Bidirectional heated, streaming electrons in the magnetosheath boundary layer, for example, from 1351 UT to 1351:10 UT, indicate that reconnection is occurring in both hemispheres, probably poleward of both the northern and southern cusps. The high ion velocities in the cusp that are primarily parallel to the magnetic field, for example, at 1343:30 UT and 1347 UT, are magnetosheath ions that have crossed the magnetopause poleward/tailward of the southern reconnection site. The ion propagation direction and the bidirectional streaming electrons indicate that the spacecraft crossed the magnetopause poleward of the high-latitude reconnection site and that the cusp field lines have one foot in the ionosphere (i.e., they are nonlobe reconnected field lines). 


\section{Cluster 3 Electrons: 9 November 2005}
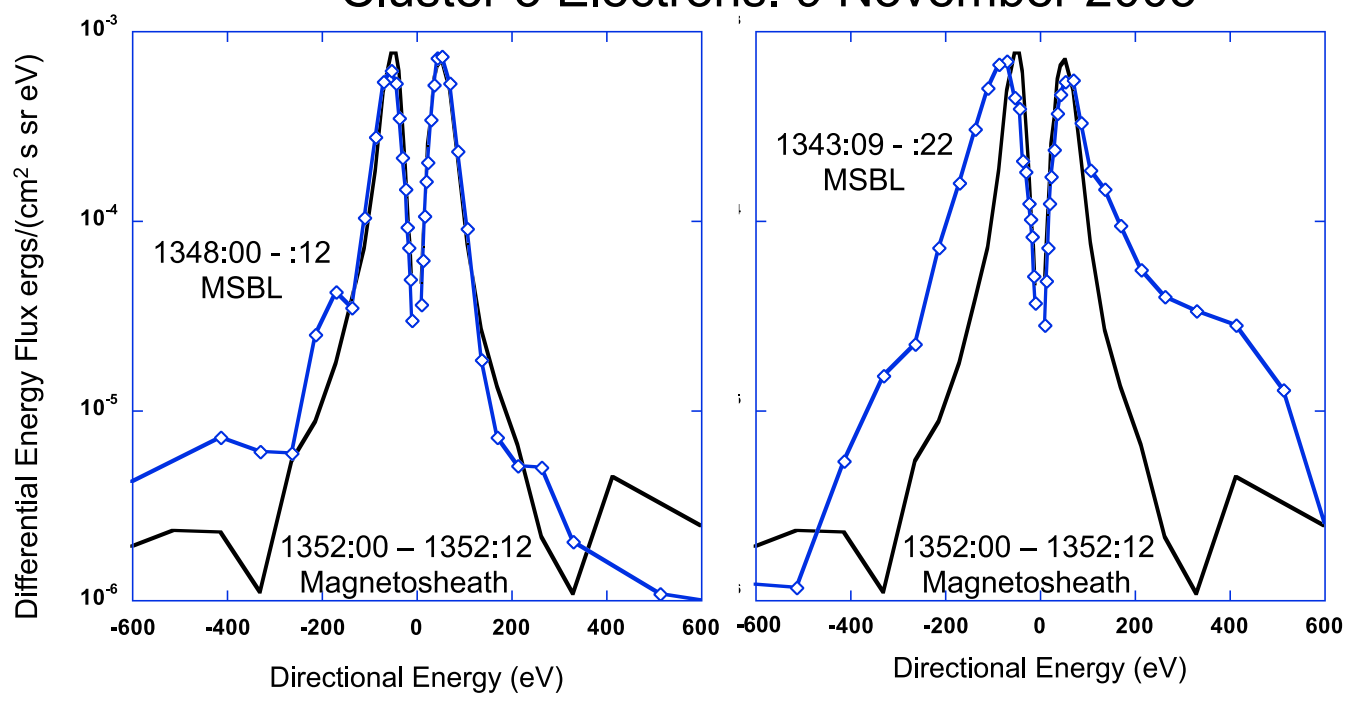

Figure 6. Similar to Figure 3, electron distributions in the magnetosheath boundary layer (MSBL) and magnetosheath. (left) A population of unidirectional electrons in the MSBL is streaming antiparallel to the magnetic field, while the populations parallel to the magnetic field in the MSBL and magnetosheath are very similar. (right) Bidirectional streaming electrons in the MSBL dominate the distribution at energies above about $100 \mathrm{eV}$.

In the magnetosheath (a) a single ion population streams antiparallel to the magnetic field, around the magnetospheric obstacle. This same magnetosheath population is in the MSBL (b), along with a second population that is also streaming antiparallel to the magnetic field. The cut along to the magnetic field in distribution (b) shows that the two populations are not completely resolved; however, the antiparallel temperature is higher and there is clearly more flux at higher negative velocities. This second population is interpreted as the combination of the "reflected" and "leaked" ion populations that are streaming out of the magnetosphere and away from the reconnection site that is equatorward/sunward of the spacecraft. In the cusp (c), there are two populations. One population is at near zero parallel velocity, while the other is streaming parallel to the magnetic field at a higher speed. The high-speed population consists of magnetosheath ions that crossed the magnetopause current layer and are propagating into the magnetosphere, but along nonlobe reconnection field lines that are connected to the northern hemisphere cusp. The origin of the distribution at near-zero velocity is not entirely clear. However, it could be the magnetosheath population that crosses the open magnetopause locally (e.g., Fuselier et al., 1995; Fuselier, Petrinec, et al., 2014). These two distributions are observed when bidirectional streaming electrons are observed. The heated, bidirectional streaming electrons are a critical piece of information that indicates that the spacecraft was either on a closed field line or, more likely, on a nonlobe reconnected field line with one foot in the northern ionosphere.

The upper left-hand panel of Figure 7 shows the field topology and the spacecraft trajectory through the reconnected field lines for the three distributions in the lower panels. The upper-right-hand panel shows the magnetic shear at the magnetopause for the crossing at 1343 UT. The format is the same as in Figure 4. For the crossing in Figure 7 at 1343 UT, the spacecraft was located at a somewhat higher latitude from the cusp than for the crossing in Figure 4. This higher latitude is compatible with the fact that the reconnection site was equatorward/sunward of the spacecraft nearly all the time in the interval in Figure 5. Also, the spacecraft appears to have no magnetic connection to the southern hemisphere antiparallel reconnection site and any magnetic connection to this site would result in ion flows parallel to the magnetic field in the MSBL. These flows would be inconsistent with the antiparallel flowing population seen in Figure 7. The lack of an apparent connection with the southern hemisphere reconnection site suggests that Cluster observes component reconnection in the southern hemisphere. Thus, there may be a mixture of component and antiparallel reconnection in this and other nonlobe reconnection events at the magnetopause.

In summary, the plasma and magnetic field data in Figures 5-7 illustrate a classic encounter of nonlobe reconnection between magnetosheath and previously closed magnetospheric field lines. The combination 

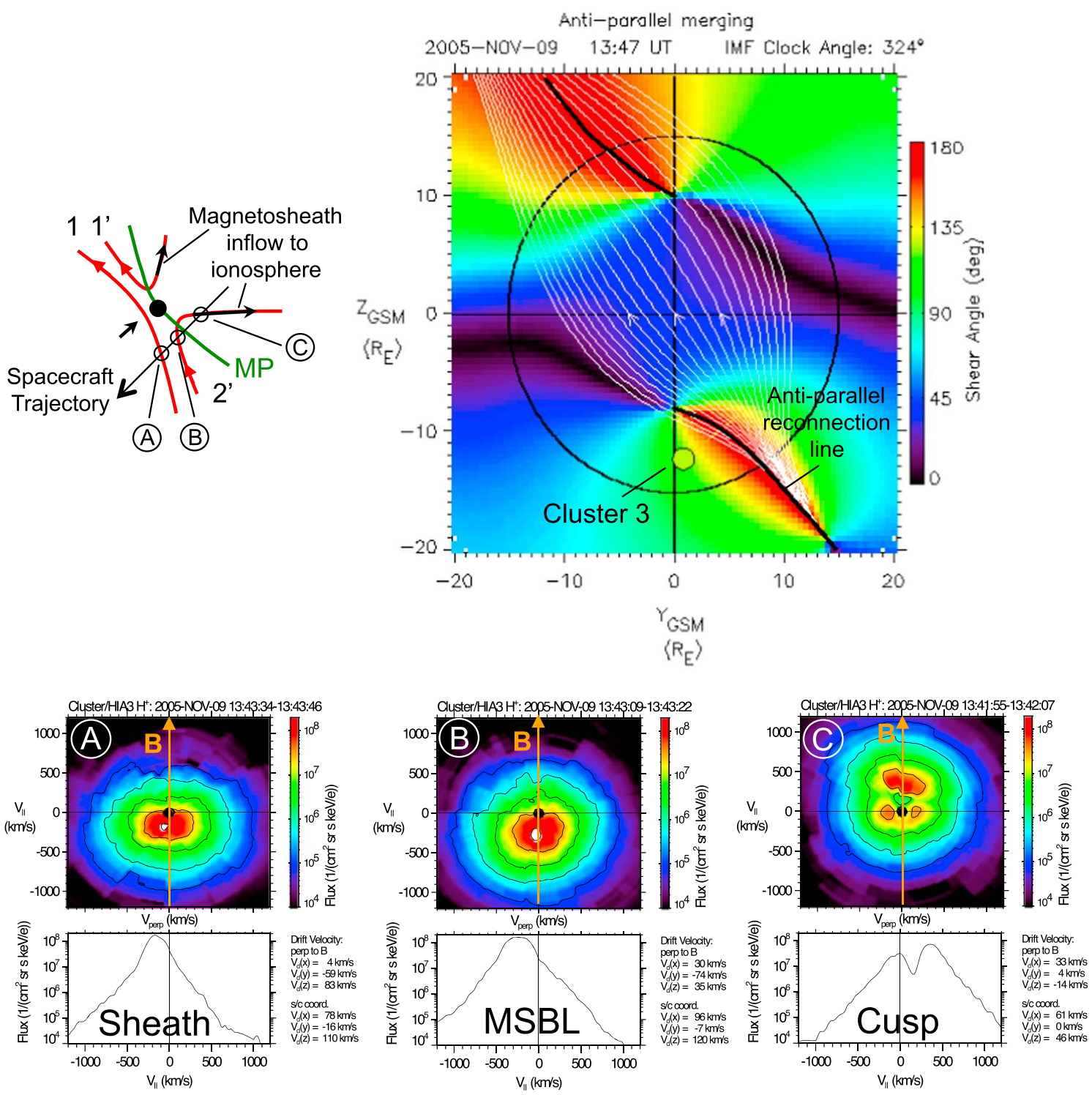

Figure 7. (upper right-hand panel) Schematic of the magnetopause crossing showing the location of the three ion distributions in the bottom panels. (upper righthand panel) $Y$-Z geocentric solar magnetospheric projection of the shear angles at the magnetopause showing the location of the Cluster spacecraft poleward of the southern cusp. The spacecraft crosses the magnetopause poleward of the southern reconnection site. In the bottom panels, the magnetosheath population is flowing antiparallel to the magnetic field, in the direction around the magnetosphere. This population is seen in the magnetosheath boundary layer (MSBL) along with a high-speed population also propagating antiparallel to the field. The two populations are not completely resolved; however, the parallel temperature of the combined populations is clearly higher than that of the magnetosheath population alone and there is considerably more flux at high velocities in the antiparallel direction in the MSBL. The antiparallel propagating population is from the reconnection site that is equatorward of the spacecraft. In the cusp, the high-speed population is propagating parallel to the field. This population is on field lines that are connected to the northern hemisphere ionosphere.

of electron and ion observations distinguishes this type of reconnection from lobe reconnection and provides the relative location of the reconnection sites and the topology of the field lines. Like the lobe reconnection example, the magnetic field, electrons, and ions play complementary roles in the interpretation of the magnetopause crossings in Figure 5. The magnetic field indicates the location of the spacecraft crossing relative to the cusp. The electrons indicate that multiple reconnection was occurring and indicate two possible interpretations where this reconnection is occurring. They also indicate that the spacecraft was never on lobe field lines in the magnetosphere. The ions help resolve which interpretation from the electrons is more likely. Finally, in combination with the magnetic field and the electron flow directions, the ion flow direction indicates that the spacecraft was on previously closed field lines in the cusp. 
For Magnetosheath to Magnetosphere Transition at High Southern Latitudes, Northward IMF

\begin{tabular}{|c|c|c|}
\hline e Cusp & $\begin{array}{l}\text { Location of S/C Crossing } \\
\text { Relative to the Southern } \\
\text { Reconnection Site }\end{array}$ & $\begin{array}{l}\text { Type of Magnetospheric Field } \\
\text { Line that Reconnects at the } \\
\text { Southern Reconnection Site }\end{array}$ \\
\hline $\begin{array}{l}\text { Poleward } \\
\text { a) } B_{\mathbf{Z}} \text { rotates north to } \\
\text { south } \\
10 \text { Mar } 2004 \\
\text { (all crossings) } \\
9 \text { Nov 2005 } \\
\text { (all crossings) }\end{array}$ & $\begin{array}{l}\text { Equatorward } \\
\text { a) MSBL: Parallel streaming } \\
\text { reflected/leaked ions } \\
10 \text { Mar } 2004 \text { (some intervals) } \\
\begin{array}{l}\text { Poleward } \\
\text { a) MSBL: Anti-parallel streaming } \\
\text { reflected/leaked ions }\end{array}\end{array}$ & $\begin{array}{l}\text { Lobe } \\
\text { a) Cusp: Anti-parallel streaming } \\
\text { magnetosheath ions } \\
\text { b) Cusp: Bi-directional streaming } \\
\text { electrons } \\
10 \text { Mar } 2004 \text { (some intervals) } \\
\text { b) Cusp: Lobe plasma } \\
10 \text { Mar } 2004 \text { (some intervals) } \\
\end{array}$ \\
\hline $\begin{array}{l}\text { Equatorward } \\
\text { a) } B_{Z} \text { remains north- } \\
\text { ward }\end{array}$ & $\begin{array}{l}\text { 9 Nov } 2005 \text { (all intervals) } \\
\text { b) MSBL: Predominantly anti-parallel } \\
\text { streaming heated electrons } \\
10 \text { Mar } 2004 \text { (some intervals) }\end{array}$ & \begin{tabular}{|l} 
Non-Lobe \\
a) Cusp: Parallel streaming \\
magnetosheath ions \\
b) Cusp: Bi-directional streaming \\
heated electrons \\
9 Nov 2005 (all intervals) \\
\end{tabular} \\
\hline
\end{tabular}

Figure 8. Flow chart that describes how to distinguish lobe and nonlobe reconnection. There are three steps to this distinction. The first two steps establish the location of the spacecraft relative to the cusp and relative to the southern hemisphere reconnection site. The last step uses the ion and electron streaming directions to distinguish crossings into the cusp that are equatorward of the reconnection site and poleward of the reconnection site for nonlobe or lobe reconnection. Magnetic field and streaming directions change for high northern latitude magnetopause crossings.

In Figure 5, the magnetic field rotation from north in the magnetosheath to south in the cusp indicates that the spacecraft always crossed the magnetopause poleward/tailward of the magnetospheric cusp. This location is also evident in the upper right-hand panel in Figure 7. Bidirectional streaming electrons in the MSBL indicate that reconnection was probably occurring poleward of both the northern and southern hemisphere cusps. The unidirectional, predominately antiparallel streaming electrons in the MSBL, combined with the parallel ion flows in the cusp indicate that the spacecraft was often poleward/tailward of the southern hemisphere reconnection site. The alternating bidirectional and unidirectional streaming electrons in the MSBL indicate that the southern hemisphere reconnection site may have been relatively near the spacecraft. The spacecraft does not observe any intervals of low-energy electrons, indicative of the lobe. Instead, the ion flow velocities in the cusp are parallel to the magnetic field, indicating crossings onto field lines that are poleward/tailward of the reconnection site. The ubiquitous presence of bidirectional streaming electrons earthward of the magnetopause indicates that at least one foot of these field lines is in the ionosphere. Thus, the combination of a crossing poleward/tailward of a reconnection site and reconnected field lines with at least one foot in the ionosphere indicates that the spacecraft observed nonlobe reconnection similar to the topology in the right-hand panel of Figure 1.

Figure 8 is a flowchart that compares and contrasts the observations from the two events in this section. Both events occur at the high-latitude southern magnetopause under northward IMF, and Figure 8 describes the interpretation of crossings from the magnetosheath to the magnetosphere. The interpretation of the observations occurs in three steps. In the first step, the location of the spacecraft crossing relative to the cusp is determined by the change in the $Z$ component of the magnetic field. The rotation of the field from northward to southward indicates that the spacecraft crosses the magnetopause poleward of the cusp for both events. In the second step, the location of the spacecraft crossing relative to the southern reconnection site is determined by the direction of propagation of the reflected/leaked ions in the MSBL and the dominant streaming direction of the electrons in the MSBL. For some MSBL intervals for the 10 Mar 2004 event, reflected/leaked ions stream parallel to the field, indicating a spacecraft location equatorward of the reconnection site. For other MSBL intervals for the 10 Mar 2004 event, there are predominantly antiparallel streaming heated electrons, indicating that the spacecraft was sometimes poleward of the reconnection site. In contrast, for all MSBL intervals for the 9 November 2005 event, reflected/leaked ions stream antiparallel to the field. The predominantly antiparallel streaming heated electrons indicate that the spacecraft was always poleward of the reconnection site. Finally, in the third step, the type of magnetospheric field line that reconnects at the southern reconnection site is determined from the ion streaming direction in the cusp and the presence or absence of lobe plasma. For the 10 March 2004 event, the antiparallel streaming ions (i.e., toward the southern ionosphere) indicate lobe field lines when the spacecraft crosses equatorward of 
the reconnection site. For those crossings poleward of the reconnection site, lobe plasma is observed (only low energy electrons). In contrast, for the 9 November 2005 event, the parallel streaming ions in the cusp combined with the bidirectional streaming, heated electrons indicate nonlobe field lines with one foot in the northern hemisphere.

\section{Survey of High-Latitude Reconnection}

Section 3 demonstrated that combined ion, electron, and field observations are able to distinguish lobe and nonlobe reconnection topologies for northward IMF. In this section, a survey of Cluster magnetopause crossings is conducted to estimate approximately how often nonlobe reconnection occurs. The starting point of this survey is a database of 6,845 magnetopause crossings by Cluster from 2001 to December 2009 that were analyzed by Fuselier et al. (2012). They imposed several criteria on this database, which are adopted here. These criteria are (1) IMF $B x / B<0.7$, to avoid problems with the draping model (Trattner et al., 2007); (2) IMF clock angle within $\pm 55^{\circ}$ of northward; and (3) magnetopause crossings within $\pm 4 \mathrm{hr}$ of local noon. Out of the original database, 388 magnetopause crossings over latitudes ranging from $+80^{\circ}$ to $-90^{\circ}$ were selected. Fuselier et al. (2012) showed that, for this database, reconnection was occurring for at least $90 \%$ of the events, in agreement with other studies of the northward IMF magnetopause. Here two additional criteria are imposed: (1) northward IMF \pm 5 min around the magnetopause crossing or crossings and (2) magnetopause crossings poleward of either the northern or southern hemisphere magnetospheric cusp. The rotation in the magnetic field from positive in the magnetosheath to negative in the magnetosphere, as seen in the crossings in Figures 2 and 5, indicates that the spacecraft crosses the magnetopause poleward of the cusp. With these two additional criteria, a large number of low-latitude crossings were eliminated and 39 magnetopause crossings poleward of the cusp that met all the criteria were identified. These 39 crossings are summarized in Table 1.

Columns in Table 1, from left to right, are the event (magnetopause crossing) number, event date, magnetopause crossing time, GSM coordinates of the Cluster 3 spacecraft in Earth Radii $\left(R_{E}\right)$, the clock angle of the IMF, the GSM latitude of the crossing, evidence for bidirectional streaming electrons in the MSBL, evidence of lobe intervals in the magnetosphere, and the reconnection type $(L=$ lobe reconnection and $\mathrm{NL}=$ nonlobe reconnection). Bidirectional streaming electrons in the MSBL are evidence for multiple reconnection sites. For lobe reconnection, these sites are located in opposite hemispheres. The presence of lobe intervals is used to indicate the possibility of lobe reconnection; however, this evidence is probably necessary, but not sufficient. Finally, the type of reconnection is determined by the following logic.

For lobe reconnection, the presence of lobe intervals in the magnetosphere near the crossing is required and either one of two flow characteristics is required: (1) antiparallel ion flows in the southern hemisphere or parallel flows in the northern hemisphere for crossings equatorward of the reconnection site or (2) either parallel electron streaming in the southern hemisphere or antiparallel electron streaming in the northern hemisphere interspersed with lobe intervals for crossings poleward/tailward of the reconnection site. Examples of these criteria were discussed for the crossings in Figure 2 and are illustrated in the flowchart in Figure 8 . If no lobe intervals were observed and the crossings were equatorward of the reconnection site, then the type of reconnection is ambiguous and identified by a question mark.

For nonlobe reconnection, identification is based on the observations of bidirectional streaming electrons in the cusp and either parallel ion streaming in the southern hemisphere or antiparallel ion streaming in the northern hemisphere for crossings poleward of the reconnection site. Examples of this criterion were discussed for the crossing in Figure 5 and illustrated in the flowchart in Figure 8.

\section{Discussion and Conclusions}

Totaling up the number of events in Table 1, there are 5 events where the type of reconnection is not known, 10 events where nonlobe reconnection was occurring, and 24 events where lobe reconnection was occurring. Thus, $30 \%$ of the total events where the type of reconnection could be identified were nonlobe reconnection events. Bidirectional streaming electrons were not observed in the MSBL in only three events. Two of these three events were nonlobe reconnection events. As pointed out in section 3, if the spacecraft is always poleward of the reconnection site, then it is possible to only observe unidirectional streaming electrons in the MSBL even though reconnection may be occurring in both cusps. Thus, it is likely that reconnection is 
Table 1

High-Latitude Magnetopause Crossings During Northward IMF

\begin{tabular}{|c|c|c|c|c|c|c|c|c|c|c|}
\hline Event \# & $\begin{array}{c}\text { Date } \\
(\mathrm{mm} / \mathrm{dd} / \mathrm{yy})\end{array}$ & $\begin{array}{l}\text { MP time } \\
\text { (hh:mm) }\end{array}$ & $\begin{array}{c}\operatorname{GSM} x \\
\left(R_{E}\right)\end{array}$ & $\begin{array}{c}\mathrm{GSM} Y \\
\left(R_{E}\right)\end{array}$ & $\begin{array}{c}\mathrm{GSM} Z \\
\left(R_{E}\right)\end{array}$ & $\begin{array}{c}\text { Clock angle } \\
\text { (deg) }\end{array}$ & $\begin{array}{l}\text { GSM lat } \\
\text { (deg) }\end{array}$ & $\begin{array}{c}\text { Evidence of } \\
\text { bidirectional streaming } \\
\text { electrons }(\mathrm{Y} \text { or } \mathrm{N})\end{array}$ & $\begin{array}{l}\text { Evidence of } \\
\text { lobe }(\mathrm{Y} \text { or } \mathrm{N})\end{array}$ & $\begin{array}{c}\text { Reconnection type } \\
(\mathrm{L}=\text { lobe, } \mathrm{NL}=\text { nonlobe, } \\
?=\text { not known) }\end{array}$ \\
\hline 1 & $12 / 03 / 01$ & $10: 03$ & 3.29 & 6.02 & -11.13 & 6 & -74 & $Y$ & $\mathrm{~N}$ & $?$ \\
\hline 2 & $12 / 03 / 01$ & $10: 14$ & 3.30 & 5.73 & -11.06 & -8 & -73 & $Y$ & $Y$ & L \\
\hline 3 & $12 / 03 / 01$ & $10: 25$ & 3.31 & 5.54 & -11.01 & 15 & -73 & $Y$ & Y & $\mathrm{L}$ \\
\hline 4 & $12 / 03 / 01$ & $10: 49$ & 3.33 & 4.70 & -10.73 & -26 & -73 & $\mathrm{Y}$ & $\mathrm{Y}$ & L \\
\hline 5 & $02 / 08 / 02$ & $02: 48$ & 5.62 & 2.30 & -8.11 & 21 & -55 & $Y$ & $\mathrm{~N}$ & $?$ \\
\hline 6 & $04 / 23 / 02$ & 08:18 & 3.99 & -1.84 & 7.38 & 36 & 62 & $Y$ & $\mathrm{~N}$ & $\mathrm{NL}$ \\
\hline 7 & $05 / 21 / 02$ & 22.08 & 3.82 & -6.67 & 5.81 & -8 & 57 & N & $\mathrm{N}$ & NL \\
\hline 8 & $12 / 19 / 02$ & $06: 54$ & 3.23 & -0.73 & -7.94 & 48 & -68 & $Y$ & $Y$ & L \\
\hline 9 & $01 / 28 / 03$ & $17: 21$ & 3.08 & -0.54 & -8.36 & 45 & -70 & $Y$ & $Y$ & L \\
\hline 10 & 03/19/03 & $15: 54$ & 1.71 & -0.78 & -9.73 & 54 & -80 & $Y$ & $Y$ & L \\
\hline 11 & $04 / 12 / 03$ & 08:03 & 2.58 & -4.51 & -11.29 & 42 & -77 & $Y$ & $\mathrm{~N}$ & NL \\
\hline 12 & $12 / 27 / 03$ & $18: 38$ & 4.21 & 2.16 & -8.84 & 12 & -65 & $Y$ & $Y$ & $\mathrm{~L}$ \\
\hline 13 & $02 / 06 / 04$ & 03:42 & 5.55 & 2.13 & -9.60 & 49 & -60 & $Y$ & $Y$ & L \\
\hline 14 & $02 / 27 / 04$ & $15: 41$ & 1.52 & -0.44 & -9.06 & 3 & -80 & $Y$ & $Y$ & L \\
\hline 15 & 03/10/04 & $12: 26$ & 1.65 & -1.63 & -9.56 & -18 & -80 & $Y$ & $Y$ & L \\
\hline 16 & $11 / 09 / 04$ & $23: 29$ & 2.97 & 0.42 & -7.67 & -38 & -69 & $Y$ & $Y$ & L \\
\hline 17 & $11 / 09 / 04$ & $23: 48$ & 3.03 & 0.06 & -7.37 & -40 & -68 & $Y$ & $\mathrm{~N}$ & $?$ \\
\hline 18 & $11 / 21 / 04$ & $16: 47$ & 2.63 & 3.78 & -11.12 & -43 & -77 & $Y$ & $Y$ & L \\
\hline 19 & $11 / 21 / 04$ & $16: 50$ & 2.63 & 3.78 & -11.12 & -43 & -77 & $Y$ & $\mathrm{~N}$ & NL \\
\hline 20 & $12 / 22 / 04$ & $17: 30$ & 3.84 & 0.77 & -8.66 & 20 & -66 & $Y$ & $Y$ & $\mathrm{~L}$ \\
\hline 21 & 01/01/05 & 03:24 & 5.63 & 3.79 & -9.50 & 16 & -59 & $Y$ & $\mathrm{~N}$ & NL \\
\hline 22 & $01 / 20 / 05$ & 03:20 & 6.78 & 3.79 & -9.52 & 3 & -55 & $Y$ & $Y$ & NL \\
\hline 23 & $01 / 20 / 05$ & $03: 23$ & 6.78 & 3.79 & -9.52 & 3 & -55 & $Y$ & $Y$ & NL \\
\hline 24 & 02/10/05 & $15: 46$ & 3.32 & -0.31 & -9.38 & -6 & -70 & $Y$ & $Y$ & L \\
\hline 25 & $04 / 22 / 05$ & $22: 03$ & 0.30 & -0.48 & -12.22 & 39 & -89 & $Y$ & $\mathrm{~N}$ & $?$ \\
\hline 26 & $11 / 02 / 05$ & $14: 24$ & 3.37 & -1.63 & -8.94 & -5 & -69 & $Y$ & $Y$ & L \\
\hline 27 & $11 / 09 / 05$ & $13: 47$ & 2.34 & 0.83 & -12.34 & -36 & -79 & $Y$ & $\mathrm{~N}$ & NL \\
\hline 28 & $11 / 09 / 05$ & $14: 32$ & 2.66 & 0.59 & -11.69 & -45 & -77 & $Y$ & $Y$ & $\mathrm{~L}$ \\
\hline 29 & $12 / 22 / 05$ & $10: 22$ & 5.13 & -0.75 & -10.46 & 31 & -64 & $Y$ & $Y$ & L \\
\hline 30 & $12 / 22 / 05$ & $11: 17$ & 4.65 & -1.58 & -9.72 & 7 & -64 & $Y$ & $Y$ & L \\
\hline 31 & $01 / 03 / 06$ & 07:47 & 5.37 & -2.78 & -10.41 & 17 & -63 & $Y$ & $Y$ & L \\
\hline 32 & $01 / 08 / 06$ & 01:05 & 6.07 & 3.87 & -10.16 & -45 & -59 & $Y$ & $\mathrm{~N}$ & $?$ \\
\hline 33 & $01 / 08 / 06$ & $01: 11$ & 6.07 & 3.87 & -10.16 & -45 & -59 & $Y$ & $\mathrm{~N}$ & $\mathrm{~L}$ \\
\hline 34 & $01 / 08 / 06$ & 01:37 & 5.70 & 3.22 & -10.13 & 40 & -61 & $Y$ & $\mathrm{~N}$ & NL \\
\hline 35 & $02 / 03 / 06$ & $06: 22$ & 4.64 & -0.37 & -10.65 & 53 & -66 & $Y$ & $\mathrm{~N}$ & $\mathrm{~L}$ \\
\hline 36 & $02 / 03 / 06$ & $06: 34$ & 4.36 & -0.61 & -10.52 & 51 & -68 & $Y$ & $Y$ & L \\
\hline 37 & $02 / 15 / 06$ & $04: 16$ & 3.54 & 0.33 & -10.74 & 25 & -72 & $\mathrm{~N}$ & $\mathrm{~N}$ & $\mathrm{NL}$ \\
\hline 38 & $02 / 15 / 16$ & $04: 33$ & 3.24 & 0.09 & -10.61 & 43 & -73 & $\mathrm{~N}$ & $Y$ & L \\
\hline 39 & 03/18/06 & 02:31 & 1.50 & 0.58 & -11.47 & 34 & -83 & $Y$ & $Y$ & L \\
\hline
\end{tabular}

Note. Nine events show evidence of nonlobe reconnection, 35 events show evidence of lobe reconnection, and 5 events are not clear.

occurring poleward of both cusps essentially all the time independent of the type of reconnection (lobe or nonlobe). As discussed in the introduction, this reconnection poleward of both cusps complicates the magnetic topology, but lobe and nonlobe reconnection in the vicinity of one of the cusps are still distinguishable.

There have been previous simulations of nonlobe reconnection at the magnetopause (e.g., Watanabe et al., 2017). In addition, there have been previous observations of nonlobe reconnection at the magnetopause. Lavraud et al. (2018) surveyed 7 years of THEMIS particle data for the simultaneous presence of high-energy magnetospheric ion and electron populations and magnetosheath energy ion and electron populations in the low latitude boundary layer during northward IMF. The high-energy populations are rapidly lost when closed field lines are open, as in nonlobe reconnection. However, if there is reconnection first in one 
hemisphere and a second reconnection of the same magnetosheath field line with a closed magnetic field line in the magnetosphere, then both the high-energy magnetospheric and lower-energy magnetosheath ion and electron populations are retained. Lavraud et al. (2018) found that only $6 \%$ of the total events fit this type of nonlobe reconnection. However, they likely underestimate the occurrence frequency of nonlobe reconnection because their observations require that the second, nonlobe reconnection occurs after the first reconnection event and that the energetic magnetospheric ion and electron populations must be present on the closed field lines prior to the second reconnection. More importantly, they had a sufficient number of events to establish that this restrictive type of nonlobe reconnection favors northward IMF when the $B_{Y}$ component is fairly large. Thus, the selection here of events with weaker $B_{Y}$ components may result in fewer nonlobe reconnection events than if the full range of northward IMF orientations was used.

The presentation of separate lobe and nonlobe reconnection observations in Figures 2 and 5 suggests that the two reconnection scenarios may be mutually exclusive. However, Onsager et al. (2001) demonstrated for a long duration encounter with the high-latitude magnetopause that both types of reconnection may occur either closely spaced in time or space. Furthermore, paired events 27 and 28 and 33 and 34 indicate that the type of reconnection changes for two separate magnetopause encounters within 45 or 20 min of one another, respectively.

Because of the complications in identifying lobe and nonlobe reconnection, the possible biasing of the sampling, and the possible simultaneous occurrence of lobe and nonlobe reconnection in extended magnetopause encounters, drawing quantitative conclusions from the survey results in Table 1 should be done very carefully. Pending a better way to survey the large data sets from Cluster and THEMIS, it is safe to say that nonlobe reconnection appears to occur frequently at the magnetopause for northward IMF. Also, the occurrence of nonlobe reconnection may be related to the length of time the IMF was northward (and the amount of open lobe field lines available for reconnection). Therefore, the trapping of flux and the convection of field lines into the Earth's magnetotail for northward IMF should be reconsidered in light of the strong possibility that nonlobe reconnection occurs frequently at the magnetopause.

\section{Acknowledgments}

This research was funded by NASA grant NNX09AM72G. Cluster data are accessible via the Cluster and Double Star Science Archive at https://www. cosmos.esa.int/web/csa. Work at IRAP was supported by CNRS and CNES

\section{References}

Badman, S. V., Master, A., Hasegawa, H., Fujimoto, M., Radioti, A., Grodent, D., et al. (2013). Bursty magnetic reconnection at Saturn's magnetopause. Geophysical Research Letters, 40, 1027-1031. https://doi.org/10.1002/grl.50199

Balogh, A., Dunlop, M. W., Cowley, S. W. H., Southwood, D. J., Thomlinson, J. G., \& the Cluster magnetometer team (1997). The Cluster magnetic field investigation. Space Science Reviews, 79, 65.

Broll, J. M., Fuselier, S. A., \& Trattner, K. J. (2017). Locating dayside magnetopause reconnection with exhaust ion distributions. Journal of Geophysical Research: Space Physics, 122, 5105-5113. https://doi.org/10.1002/2016JA023590

Cowley, S. W. H. (1982). The causes of convection in the Earth's magnetosphere: A review of developments during the IMS. Reviews of Geophysics and Space Physics, 20(3), 531-565. https://doi.org/10.1029/RG020i003p00531

Cowley, S. W. H. (1983). Interpretation of observed relations between solar wind characteristics and effects at ionospheric altitudes. In B. Hultquist, \& T. Hagfors (Eds.), High latitude space plasma physics (pp. 225-249). New York: Springer.

Dungey, J. W. (1963). The structure of the exosphere or adventures in velocity space. In C. De Witt, J. Hieblot \& L. Le Beau (Eds.), Geophysics, the Earth's environment (pp. 503-560). New York: Gordon and Breach.

Ebert, R. W., Allegrini, F., Bagenal, F., Bolton, S. J., Connerney, J. E. P., Clark, G., et al. (2017). Accelerated flows at Jupiter's magnetopause: Evidence for magnetic reconnection along the dawn flank. Geophysical Research Letters, 44, 4401-4409. https://doi.org/10.1002/ 2016GL072187

Fuselier, S. A., Anderson, B. J., \& Onsager, T. G. (1995). Particle signatures of magnetic topology at the magnetopause: AMPTE/CCE observations. Journal of Geophysical Research, 100(A7), 11,805-11,822. https://doi.org/10.1029/94JA02811

Fuselier, S. A., Anderson, B. J., \& Onsager, T. G. (1997). Electron and ion signatures of field line topology at the low shear magnetopause. Journal of Geophysical Research, 102(A3), 4847-4863. https://doi.org/10.1029/96JA03635

Fuselier, S. A., Frahm, R., Lewis, W. S., Masters, A., Mukherjee, J., Petrinec, S. M., \& Sillanpaa, I. J. (2014). The location of magnetic reconnection at Saturn's magnetopause-A comparison with Earth. Journal of Geophysical Research: Space Physics, 119, 2563-2578. https://doi.org/ 10.1002/2013JA019684

Fuselier, S. A., Klumpar, D. M., \& Shelley, E. G. (1991). Ion reflection and transmission during reconnection at the Earth's subsolar magnetopause. Geophysical Research Letters, 18(2), 139-142. https://doi.org/10.1029/90GL02676

Fuselier, S. A., Petrinec, S. M., Trattner, K. J., \& Lavraud, B. (2014). Magnetic field topology for northward IMF reconnection: lon observations. Journal of Geophysical Research: Space Physics, 119, 9051-9071. https://doi.org/10.1002/2014JA020351

Fuselier, S. A., Trattner, K. J., Petrinec, S. M., \& Lavraud, B. (2012). Dayside magnetic topology at the Earth's magnetopause for northward IMF. Journal of Geophysical Research, 117, A08235. https://doi.org/10.1029/2012JA017852

Gosling, J. T., Thomsen, M. F., Bame, S. J., Elphic, R. C., \& Russell, C. T. (1991). Observations of reconnection of interplanetary and lobe magnetic field lines at the high-latitude magnetopause. Journal of Geophysical Research, 96(A8), 14,097-14,106. https://doi.org/10.1029/91JA01139

Johnstone, A. D., Alsop, C., Burge, S., Carter, P. J., Coates, A. J., Coker, A. J., et al. (1997). PEACE: A plasma electron and current experiment. Space Science Reviews, 79(1/2), 351-398. https://doi.org/10.1023/A:1004938001388

Lavraud, B., Fedorov, A., Budnik, E., Thomsen, M. F., Grigoriev, A., Cargill, P. J., et al. (2005). High-altitude cusp flows dependence on IMF orientation: A three-year Cluster statistical study. Journal of Geophysical Research, 110, A02209. https://doi.org/10.1029/2004JA010804 
Lavraud, B., Jacquey, C., Achilli, T., Fuselier, S. A., Grigorenko, E., Phan, T. D., et al. (2018). Concomitant double ion and electron populations in the Earth's magnetopause boundary layers from double reconnection with lobe and closed field lines. Journal of Geophysical Research: Space Physics, 123, 5407-5419. https://doi.org/10.1029/2017JA025152

Lavraud, B., Thomsen, M. F., Lefebvre, B., Schwartz, S. J., Seki, K., Phan, T. D., et al. (2006). Evidence for newly closed magnetosheath field lines at the dayside magnetopause under northward IMF. Journal of Geophysical Research, 11, A05211. https://doi.org/10.1029/2005JA011266

Lavraud, B., Thomsen, M. F., Taylor, M. G. G. T., Wang, Y. L., Phan, T. D., Schwartz, S. J., et al. (2005). Characteristics of the magnetosheath electron boundary layer under northward interplanetary magnetic field: Implications for high-latitude reconnection. Journal of Geophysical Research, 110, A06209. https://doi.org/10.1029/2004JA010808

Lepping, R. P., Acũna, M. H., Burlaga, L. F., Farrell, W. M., Slavin, J. A., Schatten, K. H., et al. (1995). The Wind magnetic field instrument. In C. T. Russell (Ed.), The global geospace mission (pp. 207-229). The Netherlands: Kluwer Academic Press.

McAndrews, H. J., Owen, C. J., Thomsen, M. F., Lavraud, B., Coates, A. J., Dougherty, M. K., \& Young, D. T. (2008). Evidence for reconnection at Saturn's magnetopause. Journal of Geophysical Research, 113, A04210. https://doi.org/10.1029/2007JA012581

McComas, D. J., Bame, S. J., Barker, P., Feldman, W. C., Phillips, J. L., Riley, P., \& Griffee, J. W. (1998). Solar Wind Electron Proton Alpha Monitor (SWEPAM) for the Advanced Composition Explorer. Space Science Reviews, 86, 563-612.

Ogilvie, K. W., Chornay, D. J., Fritzenreiter, R. J., Hunsaker, F., Keller, J., Lobell, J., et al. (1995). SWE: A comprehensive plasma instrument for the Wind spacecraft. In C. T. Russell (Ed.), The global geospace mission (pp. 55-77). Norwell, MA: Kluwer Academic Press.

Onsager, T. G., Scudder, J. D., Lockwood, M., \& Russell, C. T. (2001). Reconnection at the high-latitude magnetopause during northward interplanetary magnetic field conditions. Journal of Geophysical Research, 106(A11), 25,467-25,488. https://doi.org/10.1029/ 2000JA000444

Reme, H., Aoustin, C., Bosqued, J. M., Dandouras, I., Lavraud, B., Sauvaud, J. A., et al. (2001). First multispacecraft ion measurements in and near the Earth's magnetosphere with the identical Cluster ion spectrometry (CIS) experiment. Annales Geophysicae, 19(10/12), 1303-1354 https://doi.org/10.5194/angeo-19-1303-2001

Smith, C. W., Acuña, M. H., Burlaga, L. F., L'Heureux, J., Ness, N. F., \& Scheifele, J. (1998). The ACE magnetic field experiment. Space Science Reviews, 86, 613-632.

Szita, S., Fazakerley, A. N., Carter, P. J., James, A. M., Travnicek, P., Watson, G., et al. (2001). Cluster PEACE observations of electrons of spacecraft origin. Annales de Geophysique, 19(10/12), 1721-1730. https://doi.org/10.5194/angeo-19-1721-2001

Trattner, K. J., Mulcock, J. S., Petrinec, S. M., \& Fuselier, S. A. (2007). Probing the boundary between antiparallel and component reconnection during southward interplanetary magnetic field conditions. Journal of Geophysical Research, 112, A08210. https://doi.org/10.1029/ 2007JA012270

Vennerstrom, S., Moretto, T., Rastätter, L., \& Raeder, J. (2005). Field-aligned currents during northward interplanetary magnetic field: Morphology and causes. Journal of Geophysical Research, 110, A06205. https://doi.org/10.1029/2004JA010802

Watanabe, M., Fujita, S., Tanaka, T., Kubota, Y., Shinagawa, H., \& Murata, K. T. (2017). A magnetohydrodynamic modeling of the interchange cycle for oblique northward interplanetary magnetic field. Journal of Geophysical Research: Space Physics, 123, 272-286. https://doi.org/ 10.1002/2017JA024468

Zhong, J., Pu, Z. Y., Dunlop, M. W., Bogdanova, Y. V., Wang, X. G., Xiao, C. J., et al. (2013). Three-dimensional magnetic flux rope structure formed by multiple sequential X-line reconnection at the magnetopause. Journal of Geophysical Research: Space Physics, 118, 1904-1911. https://doi.org/10.1002/jgra.50281 